\title{
Targeted axillary dissection with preoperative tattooing of biopsied positive axillary lymph nodes in breast cancer
}

\author{
J. GATEK ${ }^{1,2}$, V. PETRU 1 , P. KOSAC ${ }^{1}$, M. RATAJSKY ${ }^{1}$, J. DUBEN ${ }^{1}$, B. DUDESEK ${ }^{1}$, P. JANCIK ${ }^{1}$, M. ZABOJNIKOVA ${ }^{3}$, J. KATRUSAK ${ }^{4}$, P. OPELOVA ${ }^{4}$, \\ L. PODRAZSKA ${ }^{4}$, P. VAZAN 5 , P. KUDLOVA², P. HOLIK ${ }^{2}$, B. MELICHAR ${ }^{6}$, D. VRANA ${ }^{7, *}$
}

${ }^{1}$ Department of Surgery, EUC Clinic Zlin, Zlin, Czech Republic; ${ }^{2}$ Tomas Bata University in Zlin, Zlin, Czech Republic; ${ }^{3}$ Department of Oncology, Tomas Bata Hospital, Zlin, Czech Republic; ${ }^{4}$ Department of Radiology, EUC Clinic Zlin, Zlin, Czech Republic; ${ }^{5}$ CGB Laboratory, Tomas Bata University in Zlin, Zlin, Czech Republic; ${ }^{6}$ Department of Oncology, Faculty of Medicine and Dentistry, Palacky University Olomouc, Olomouc, Czech Republic; ' Department of Oncology and Radiotherapy, Comprehensive Cancer Centre, Novy Jicin, Czech Republic

${ }^{*}$ Correspondence: davvrana@gmail.com

Received December 28, 2019 / Accepted February 24, 2020

\begin{abstract}
Sentinel lymph node biopsy (SLNB) has emerged as an alternative to axillary lymph node dissection during breast cancer surgery during the last 2 decades. However, there are several controversies regarding the indication of the sentinel node biopsy after neoadjuvant chemotherapy which can convert positive lymph nodes to negative. The false-negative rate after neoadjuvant chemotherapy is unacceptably high. This high false-negative rate can be decreased by marking of the positive lymph nodes and removal during sentinel lymph node biopsy procedure in addition to the sentinel lymph nodes. The aim of this study was to investigate the possibility of carbon tattooing of the positive sentinel lymph nodes before neoadjuvant chemotherapy. In 2016, a prospective protocol was launched investigating the black carbon tattooing procedure of the suspective and positive axillary lymph nodes by injecting 0.1-0.5 carbon ink in normal saline under ultrasound guidance. All patients underwent black carbon tattooing of the suspected or positive axillary lymph nodes before the chemotherapy or one week before the primary surgery when chemotherapy was not indicated in the neoadjuvant setting. Sentinel lymph nodes together with lymph nodes marked by the black carbon ink were removed and histologically evaluated. So far 27 patients were treated under this protocol. Breast saving surgery was performed in 22 cases and mastectomy in 5 cases. All patients had invasive ductal carcinoma. In 20 patients neoadjuvant chemotherapy was indicated and in 7 patients primary surgery was performed. All lymph nodes marked by black carbon ink were successfully identified and removed. Sentinel lymph node biopsy was performed in 8 cases and sentinel lymph node biopsy followed by axillary dissection in 15 cases. Axillary dissection alone was performed in 4 cases. In 19 cases, the black carbon ink was present in the sentinel lymph node at the same time and in 4 cases carbon dye was present in other lymph nodes than the lymph node identified during SLNB, which corresponds to $17.4 \%$. In the group of patients undergoing primary surgery, in one case from six, the sentinel lymph node was negative and the lymph node marked with carbon ink positive which represents false-negative lymph node and failure of the SLNB procedure. After neoadjuvant chemotherapy, there was no false-negative lymph node identified, but the conversion of the positive lymph nodes to negative was present in 10 cases (50\%). There were no complications attributed to carbon ink tattooing. The results of positive sentinel lymph nodes tattooing have confirmed that this method is safe and allows a decrease in the false negativity rate during the sentinel node biopsy procedure.
\end{abstract}

Key words: breast cancer, neoadjuvant chemotherapy, sentinel node biopsy, tattooing node

Breast cancer represents the most common malignant disorder among women. Alone in the Czech Republic, there are 6,000 new cases diagnosed each year [1]. The tumor commonly spreads from the primary site in the breast into the ipsilateral axillary lymph nodes. The axillary lymph nodes evaluation is for many years the principal prognostic parameter. The presence of the tumor in the axillary lymph nodes has a significant impact on patient prognosis and further management regarding the systemic chemotherapy and hormonal treatment as well as radiotherapy [2, 3]. For decades, axillary dissection was a component of breast cancer surgery for malignant disease exploring the presence of metastases in axillary lymph nodes. However, axillary dissection is associated with significant morbidity that affects the quality of life of patients and the necessity of this procedure is questionable. Sentinel node biopsy markedly reduced the number of axillary dissections [4]. The results of ACOSOG Z0011 are a further step in the direction towards 
a mini-invasive approach, which further reduces the number of axillary dissections. The patients with clinically negative axillary lymph nodes are directed to SLNB and in the case of 1-2 positive lymph nodes, the axillary dissection is not performed $[5,6]$. In the case of clinically suspected axillary lymph nodes, the biopsy of such nodes is performed and in the case of positive finding, the patients are proceeding with neoadjuvant treatment. In the case of clinically negative lymph nodes before and after neoadjuvant treatment, a sentinel node biopsy is performed [7]. In patients with positive axillary lymph nodes before the neoadjuvant treatment, the ACOSOG Z0071 as well as SENTINA trial have confirmed a high rate of false-negative sentinel lymph nodes exceeding $10 \%$. In patients with positive axillary lymph nodes, the axillary dissection was indicated as standard procedure $[8,9]$. The false-negative rate decreased below $10 \%$ only when dissection of more than 3 lymph nodes and simultaneous dual lymph node marking were performed $[10,11]$. However, pathologic complete response rate after the neoadjuvant treatment in the case of triple-negative or HER2 positive tumors reaches up to $60 \%$ and this remission rate applies also to axillary lymph nodes [7]. In ACOSOG Z0071 trial, there was no requirement of positive lymph node marking with the clip before the neoadjuvant treatment, but in the situation when the marking of such nodes with the clip was performed and this node was not a sentinel lymph node, there was a further significant decrease in the falsenegative sentinel node rate. In such cases, the rate of falsenegative sentinel node dropped under $10 \%$ which is considered acceptable [12]. The use of radioactive seed for marking positive axillary lymph node led to a drop of sentinel node false-negative rate to $1.4 \%$ [13]. There are more techniques on how to mark the clinically positive axillary lymph nodes,

Table 1. SLNB after neoadjuvant chemotherapy.

\begin{tabular}{ll}
\hline SLN before neodjuvant chemotherapy & $\mathbf{2 0}$ \\
Positive node & 18 \\
Suspicious node & 2 \\
SLNB & $\mathbf{2 0}$ \\
SLN simultaneous with carbon dye node & $\mathbf{1 4}$ \\
SLN simultaneous with carbon dye node negative & 9 \\
SLN simultaneous with carbon dye node positive & 5 \\
SLN separately from carbon & $\mathbf{3}$ \\
SLN positive, carbon dye negative & 1 \\
SLN negative, carbon dye negative & 2 \\
ALND & $\mathbf{3}$ \\
Axillary nodes negative & 2 \\
Carbon dye node positive & 1 \\
Conversion positive node into negative node & \\
Conversion positive SLN into negative node & 7 \\
Scar in suspicious node after neoadjuvant chemotherapy & 1 \\
Conversion positive node into negative in ALND & \\
Carbon dye positive node into negative nodes & 2 \\
\hline
\end{tabular}

including wire, clip, or carbon ink [14]. The Stanford group has presented the experience with marking of the lymph nodes with carbon ink. The carbon ink marking has several advantages, including the price, availability, and simplicity of use $[15,16]$. This approach is inspired by the utilization in gastroenterology [17]. The aim of this study is to investigate the possibility of carbon ink marking of the metastatic axillary lymph nodes before the breast cancer surgery, and before and after neoadjuvant systemic treatment.

\section{Patients and methods}

Marking before surgery. Starting in 2016, we have launched this prospective protocol of marking suspected and positive axillary lymph nodes with $0.5-1 \mathrm{ml}$ of $4 \%$ carbon ink in normal saline. The carbon ink was injected before surgery into the subcortical zone of the lymph nodes under ultrasound control. In positive lymph nodes and in patients indicated for the primary surgical procedure, the marking was performed one week before the surgery. In patients indicated for neoadjuvant treatment, the marking was performed before the first chemotherapy cycle (Table 1).

Node identification during the surgery. The sentinel node biopsy was performed in the EUC clinic since 1998. For this procedure, the 2-day protocol is used using $100 \mathrm{MqB}$ radiocoloid, which is injected peritumoraly. Before the surgery, $1 \mathrm{ml}$ of Patentblue is also injected subarteoraly. For the identifications of lymph nodes, C-track and Gamma camera are used (Figures 1A, 1B). Blue-colored and radioactive sentinel lymph nodes are histologically evaluated. At the same time, the lymph node marked with carbon ink was also evaluated (Figures 1C, 1D). Removed lymph nodes were investigated during surgery. If at least three lymph nodes were negative after neoadjuvant treatment, including the lymph node marked with carbon ink, the axillary dissection was omitted. The carbon ink marker was always identified in the lymph node during the pathologic evaluation. Also, there was no problem to differentiate the lymph nodes marked with Patentblue as well as with carbon ink, colors were clearly differentiable.

Histopathologic lymph node evaluation. The black carbon dye-marked lymph node is considered as the lymph node with a macroscopic remarkable black pigmentation or with the histologic picture of the presence of this black dye in the lymph node capsule, in the subscapular zone, or node parenchyma. Such a node was further evaluated as a sentinel lymph node. Sections of $2 \mathrm{~mm}$ thickness of this lymph node were performed from which microtome cuts were prepared in three levels in 50 micrometers intervals. At every level, 2 cuts were obtained, one stained with $\mathrm{H}-\mathrm{E}$, and the second one stained with immunohistochemistry for cytokeratin AE1/ AE3. Black tattooing/pigmentations is usually not complete, is focal and also locally causing nonspecific granulomatous reaction of different grade, but this has no negative impact on histologic lymph node examination. In the case of any doubt, there is always a possibility to complete the basic staining 

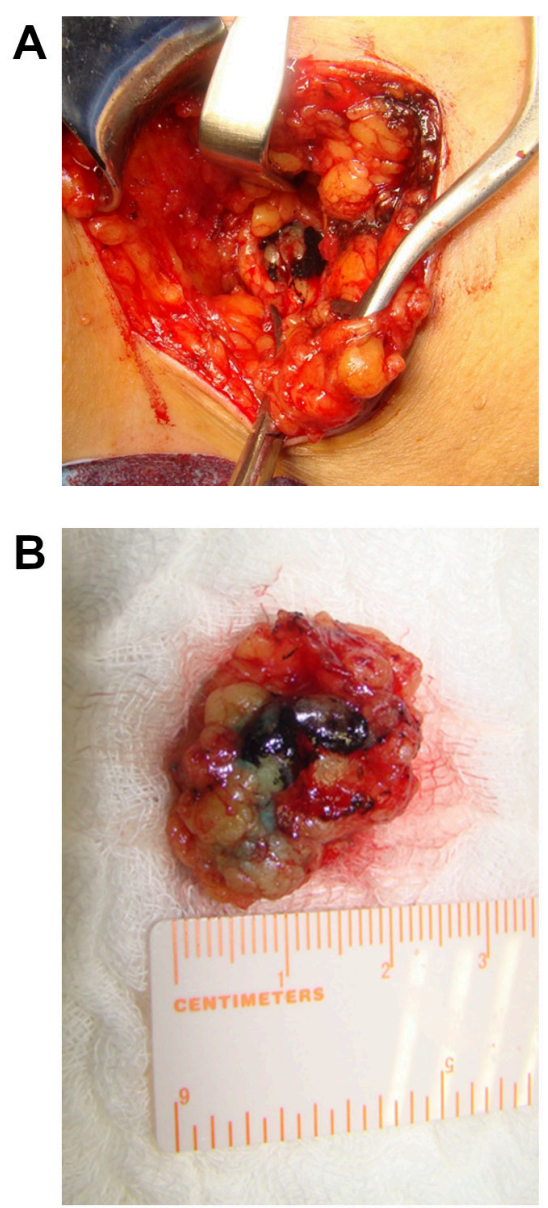

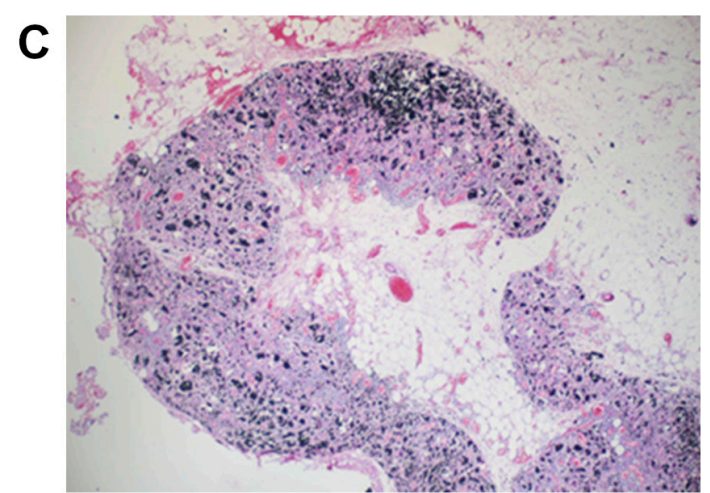

D

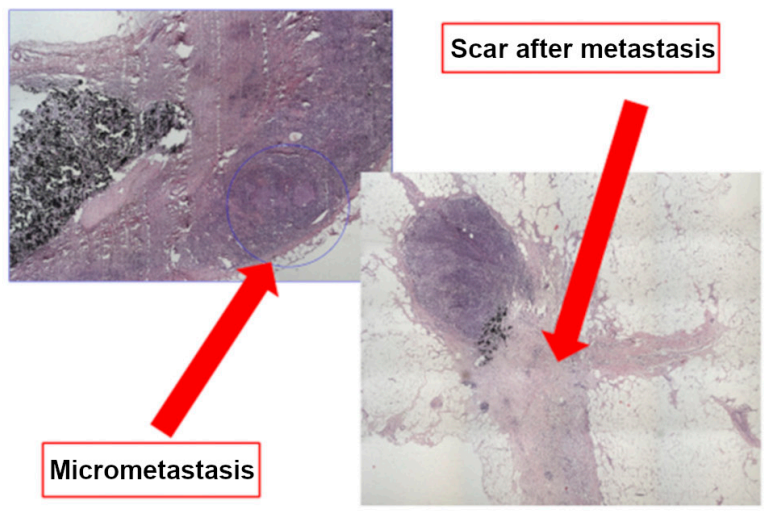

Figure 1. Node identification during the surgery. A) Intraoperative axillary carbon dye node. B) Excised sentinel node simultaneous with carbon dye. C) Carbon dye in the axillary node. D) Histologic findings after neoadjuvant chemotherapy.

with immunochemistry (cytokeratin). A possible presence of the dye in the surrounding fat tissue, which should not be confused for lymph node, should also be considered.

Neoadjuvant chemotherapy. For the neoadjuvant chemotherapy, the dose-dense combination regimen of doxorubicin/cyclophosphamide followed by weekly paclitaxel was used. In the case of HER2 positivity, trastuzumab was started with paclitaxel administration and continued after the surgery until 1 year of treatment was completed.

\section{Results}

This prospective protocol was launched in 2016 and so far 27 patients have undergone this procedure. The mean age of the patients was 56.6 years. Breast saving surgery and mastectomy were performed in 22 and 5 cases, respectively. In all patients, invasive ductal carcinoma (NST) was histologically verified. The pathologic characteristics are presented in Table 2. All lymph nodes marked with carbon ink were successfully identified and evaluated. SLNB was performed in 8 cases, SLNB followed by axillary dissection in 15 cases, and axillary dissection after carbon dye marking without SLNB in 4 cases (Tables 1, 3, 4). SLNB in simultaneous carbon dye presence was in 19 cases and carbon dye in the other lymph node was diagnosed in 4 cases (17.4\%). After the neoadjuvant chemotherapy, the sentinel lymph node (SLN) was positive and carbon ink negative in 1 case. Among patients treated with primary surgery, there was one SLN negative and carbon ink lymph node positive in one case, which represents a false-negative lymph node. The conversion of the positive lymph nodes into negative lymph nodes was observed in 10 cases. There were no complications with the carbon dye procedure. Also, the identification of the lymph nodes marked with carbon dye was easy also in the case this lymph node was a sentinel lymph node. There was also no problem distinguishing the lymph nodes marked with Patentblue and carbon dye or radiocoloid. The staining of the perinodal fat tissue with carbon dye was also observed in some cases. This perinodal staining makes the identification of the lymph nodes easier, but should not be mistaken 
Table 2. Clinicopathologic characteristic of the patients.

\begin{tabular}{|c|c|}
\hline Age & $56.6(31-76)$ \\
\hline \multicolumn{2}{|l|}{ Subtype carcinoma } \\
\hline Ductal CA (NST) & 27 \\
\hline Luminal A & 1 \\
\hline Luminal B & 11 \\
\hline Triple negative & 8 \\
\hline Her2+ & 7 \\
\hline \multicolumn{2}{|l|}{ Surgery after neoadjuvant chemotherapy } \\
\hline After neoadjuvant chemotherapy & 20 \\
\hline \multicolumn{2}{|l|}{ Response } \\
\hline Chevalier 1 & 7 \\
\hline Chevalier 2 & 3 \\
\hline Chevalier 3 & 9 \\
\hline Chevalier 4 & 1 \\
\hline Without neoadjuvant chemotherapy & 7 \\
\hline \multicolumn{2}{|l|}{ Surgery on breast } \\
\hline Conservative surgery & 22 \\
\hline Mastectomy & 5 \\
\hline \multicolumn{2}{|l|}{ Surgery in axilla } \\
\hline SLNB & 23 \\
\hline SLNB+ALND & 15 \\
\hline SLNB only & 8 \\
\hline ALDN & 4 \\
\hline \multicolumn{2}{|l|}{ Nodes } \\
\hline all nodes & 221 \\
\hline Number SNL & 56 \\
\hline SNL negative & 42 \\
\hline SNL positive & 14 \\
\hline Nonsentinel nodes & 165 \\
\hline Nonsentinel nodes negative & 115 \\
\hline Nonsentinel nodes positive & 50 \\
\hline \multicolumn{2}{|l|}{ Carbo dye nodes } \\
\hline Mean time from application carbon dye & 164 days $(8-240)$ \\
\hline SLN simultaneous with carbon dye & 19 \\
\hline SLN separately from carbon & 4 \\
\hline \multicolumn{2}{|c|}{ Conversion positive node into negative node } \\
\hline SLNB & 8 \\
\hline ALND & 2 \\
\hline
\end{tabular}

Table 3. SLNB before primary surgery.

\begin{tabular}{ll}
\hline SLN before primary surgery & 7 \\
Positive node & 6 \\
Negative node & 1 \\
SLNB & $\mathbf{6}$ \\
SLN simultaneous with carbon dye node positive & 5 \\
SLN negative carbon dye node positive & 1 \\
ALND & $\mathbf{1}$ \\
Carbon dye node positive & 1 \\
\hline
\end{tabular}

for the real lymph node. The average time from carbon ink application until the surgical procedure was 164 days. The longest interval from carbon ink application until surgery was 240 days.
Table 4. Total number of the axillary nodes.

\begin{tabular}{lcc}
\hline & $\begin{array}{c}\text { Neoadjuvant } \\
\text { chemotherapy }\end{array}$ & Primary surgery \\
\hline SLN nodes & $\mathbf{5 6}$ & $\mathbf{1 0}$ \\
Negative & & 3 \\
Positive & 7 & 7 \\
Nonsentinel nodes & $\mathbf{7 8}$ & $\mathbf{5 4}$ \\
Negative & 66 & 21 \\
Positive & 12 & 33 \\
ALND & $\mathbf{3 0}$ & $\mathbf{1 4}$ \\
Negative & 17 & 8 \\
Positive & 13 & 6 \\
Total & $\mathbf{1 6 4}$ & $\mathbf{7 8}$ \\
\hline
\end{tabular}

\section{Discussion}

Axillary lymph node status is a crucial prognostic parameter, but axillary dissection is associated with a number of complications, which can significantly affect the quality of life. A mini-invasive approach was developed with the aim to further decrease the number of axillary dissections [2, 3]. The implementation of the results of the Z0011 trial has resulted in a rapid decrease of the number of axillary dissections [4-6]. However, surgical management after neoadjuvant chemotherapy represents a specific situation. SLNB after neoadjuvant chemotherapy is a generally accepted approach in patients with negative axillary nodes before the initiation of treatment. In patients with positive lymph node before neoadjuvant therapy, the axillary dissection is generally recommended due to the high rate of a complete response. The Z0071 and SENTINA trials have confirmed an increased rate of false-negative findings after neoadjuvant chemotherapy but this rather high rate can be decreased if 3 or more lymph nodes are removed, by using dual marking and by marking the positive nodes before chemotherapy and the removal [10-11]. If axillary lymph nodes are negative and there are 3 and more histologically negative lymph nodes, MSKCC is not following with axillary dissection [18]. This approach was confirmed during St. Galen 2019 [9]. The approach used in the MD Anderson Cancer Center is to mark the positive axillary lymph nodes by the radioactive clip, performing SLNB, and removing simultaneously the marked lymph node. Using this procedure, the false-negative rate can be decreased to $1.4 \%$ [13]. For positive lymph nodes identification, the clips can be used. The identification of the marked lymph node during the surgery procedure is difficult. Therefore, an identification using the wire is usually performed which represents two procedures. Marking using the magnetic seed is expensive, requires specific instruments and equipment. In the case using of radioactive seeds for lymph node identification, it is necessary to follow the requirements for usage of the radioactive substances which represent significant limitation [14]. All these drawbacks are avoided using the carbon dye $[15,16,19]$. Carbon dye has 
been used for a long time in gastroenterology for marking of intestinal lesions. It was confirmed that the carbon dye is not resorbed, results in no wound complications, and has an acceptable price [17]. In this patient population, carbon dye was used because of the accessibility and price. Carbon dye is injected into the suspected or metastatic lymph nodes which are removed during the surgical procedure. In agreement with other studies, there was no problem with the identification of the lymph node marked with the carbon dye, also if the sentinel lymph node was involved. The interval between the carbon dye injection and surgical procedure has not affected the perioperative identification of the lymph node. The longest interval between the application and surgery was 240 days. The present study has confirmed the data reported earlier. In agreement with the Z0071 trial, we have also observed that not all sentinel lymph nodes possess carbon dye [12]. In the present study it was 17.4\%, in Z0071 trial $24 \%$ and in the series reported by Yonsei University 25\% [19]. In Stanford trial, the sentinel node and carbon ink correlated in $98 \%$ of cases, and this was explained by the fact that all patients were operated by the same surgeon [16]. In the present study with a single surgeon performing all procedures, $100 \%$ concordance rate was not reached. The number of false-negative lymph nodes has dropped to $5 \%$ in the Z0071 trial which is considered acceptable. The conversion of positive to negative nodes was observed in 10 cases, representing $50 \%$ of patients treated with neoadjuvant chemotherapy. During the histological examination, scarring of the previously positive lymph nodes was observed which suggests pathological complete response. In the case of 3 or more negative lymph nodes including the lymph node with carbon dye, the axillary dissection was omitted. If the lymph nodes were positive for any amount of tumor tissue or the number was not sufficient, the axillary dissection has followed, in accordance with St. Galen recommendations and also with results of the MSKCC experience [9, 18]. The follow-up after the surgery is still limited, but no case of axillary recurrence was observed so far. The axillary lymph node examination before the surgery consisted of an ultrasound investigation which has several limitations and requires further improvement. The management of patients, in whom the lymph node status is not histologically verified before chemotherapy, should be investigated in further prospective trials. The results of the Z0011 trial suggest the omission of the axillary dissection in patients with 1-2 positive lymph nodes [5, 6]. Marking before the surgical procedure decreases the rate of false-negative lymph nodes, which was observed in 1 case from 7 patients in the present study. Tattooing of the suspected lymph nodes in patients not treated with neoadjuvant chemotherapy further extends the utilization of this method.

In conclusion, this study has confirmed that axillary lymph node tattooing is a safe method resulting in a decreased number of false-negative lymph nodes during the sentinel node biopsy procedure.

\section{References}

[1] Dusek L, Muzik J, Maluskova D, Majek O, Pavlik T et al. [Epidemiology of Screening-Targeted Cancers According to New Data of the Czech National Cancer Registry]. Klin Onkol 2014; 27 Suppl 2: 19-39.

[2] LYMAN GH, GIULIANO AE, SOMERFIELD MR, BENSON AB 3RD, BODURKA DC et al. American Society of Clinical Oncology guideline recommendations for sentinel lymph node biopsy in early-stage breast cancer. J Clin Oncol 2005; 23: 7703-7720. https://doi.org/10.1200/ JCO.2005.08.001

[3] BEEK MA, VERHEUVEL NC, LUITEN T, KLOMPENHOUWER EG, RUTTEN HJT et al. Two decades of axillary management in breast cancer. Br J Surg 2015; 102: 16581664. https://doi.org/10.1002/bjs.9955

[4] KRAG DN, ANDERSON SJ, JULIAN TB, BROWN AM, HARLOW SP et al. Sentinel-lymph-node resection compared with conventional axillary-lymph-node dissection in clinically node-negative patients with breast cancer: overall survival findings from the NSABP B-32 randomised phase 3 trial. Lancet Oncol 2010; 11: 927-933. https://doi. org/10.1016/S1470-2045(10)70207-2

[5] CAUDLE AB, HUNT K, KUERER HM, MERIC-BERNSTAM F, LUCCI A et al. Multidisciplinary Considerations in the Implementation of the Findings from the American College of Surgeons Oncology Group (ACOSOG) Z0011 Study: A Practice-Changing Trial. Ann Surg Oncol 2011; 18: 2407-2412. https://doi.org/10.1245/s10434-011-1593-7.

[6] DELPECH Y, BRICOU A, LOUSQUY R, HUDRY D, JANKOWSKI $\mathrm{C}$ et al. The exportability of the ACOSOG Z0011 criteria for omitting axillary lymph node dissection after positive sentinel lymph node biopsy findings: a multicenter study. Ann Surg Oncol 2013; 20: 2556-2561. https:// doi.org/10.1245/s10434-013-2917-6

[7] KÜMMEL S, HOLTSCHMIDT J, LOIBL S. Surgical treatment of primary breast cancer in the neoadjuvant setting. Br J Surg 2014; 101: 912-924. https://doi.org/10.1002/ bjs. 9545

[8] CURIGLIANO G, BURSTEIN HJ, WINER EP, GNANT M, DUBSKY $\mathrm{P}$ et al. De-escalating and escalating treatments for early-stage breast cancer: the St. Gallen International Expert Consensus Conference on the Primary Therapy of Early Breast Cancer 2017. Ann Oncol 2017; 28: 1700-1712. https:// doi.org/10.1093/annonc/mdx308

[9] BALIC M, THOMSSEN C, WÜRSTLEIN R, GNANT M, HARBECK N. St. Gallen/Vienna 2019: A Brief Summary of the Consensus Discussion on the Optimal Primary Breast Cancer Treatment. Breast Care (Basel) 2019; 14: 103-110. https://doi.org/10.1159/000499931

[10] BOUGHEY JC, SUMAN VJ, MITTENDORF EA, AHRENDT GM, WILKE LG et al. Sentinel lymph node surgery after neoadjuvant chemotherapy in patients with node-positive breast cancer: the ACOSOG Z1071 (Alliance) clinical trial. JAMA 2013; 310: 1455-1461. https:/doi. org/10.1001/jama.2013.278932 
[11] KUEHN T, BAUERFEIND I, FEHM T, FLEIGE B, HAUSSCHILD M et al. Sentinel-lymph-node biopsy in patients with breast cancer before and after neoadjuvant chemotherapy (SENTINA): a prospective, multicentre cohort study Lancet Oncol 2013; 14: 609-618. https://doi.org/10.1016/S14702045(13)70166-9

[12] BOUGHEY JC, BALLMAN KV, LE-PETROSS HT, MCCALL LM, MITTENDORF EA et al. Identification and resection of the clipped node decreases the false negative rate of sentinel lymph node surgery in patients presenting with node positive breast cancer (T0-T4, N1-2) who receive neoadjuvant chemotherapy - results from ACOSOG Z1071 (Alliance). Ann Surg 2016; 263: 802-807. https://doi. org/10.1097/SLA.0000000000001375

[13] CAUDLE AS, YANG WT, KRISHNAMURTHY S, MITTENDORF EA, BLACK DM et al. Improved Axillary Evaluation Following Neoadjuvant Therapy for Patients With Node-Positive Breast Cancer Using Selective Evaluation of Clipped Nodes: Implementation of Targeted Axillary Dissection. J Clin Oncol 2016; 34: 1072-1078. https://doi. org/10.1200/JCO.2015.64.0094

[14] WOODS RW, CAMP MS, DURR NJ, HARVEY SC. A Review of Options for Localization of Axillary Lymph Nodes in the Treatment of Invasive Breast Cancer. Acad Radiol 2019; 26: 805-819. https://doi.org/10.1016/j.acra.2018.07.002
[15] CHOY N, LIPSON J, PORTER C, OZAWA M, KIERYN A et al. Initial results with preoperative tattooing of biopsied axillary lymph nodes and correlation to sentinel lymph nodes in breast cancer patients. Ann Surg Oncol 2015; 22: 377-382. https://doi.org/10.1245/s10434-014-4034-6

[16] PATEL R, MACKERRICHER W, TSAI J, CHOY N, LIPSON J et al. Pretreatment Tattoo Marking of Suscpicious Axillary Lymph Nodes: Realiability and Correlation with Sentinel Lymp Node. Ann Surg Oncol 2019; 26: 2452-2458. https:// doi.org/10.1245/s10434-019-07419-3

[17] ASKIN MP, WAYE JD, FIEDLER L, HARPAZ N. Tattoo of colonic neoplasms in 113 patients with a new sterile carbon compound. Gastrointest Endosc 2002; 56: 339-342. https:// doi.org/10.1016/s0016-5107(02)70035-7

[18] MOO TA, EDELWEISS M, HAJIYEVA S, STEMPEL M, RAISS $\mathrm{M}$ et al. Is Low-Volume Disease in the Sentinel Node After Neoadjuvant Chemotherapy an Indication for Axillary Dissection? Ann Surg Oncol 2018; 25: 1488-1494. https:// doi.org/10.1245/s10434-018-6429-2

[19] PARK S, KOO JS, KIM GM, SOHN J, KIM SI et al. Feasibility of Charcoal Tattooing of Cytology-Proven Metastatic Axillary Lymph Node at Diagnosis and Sentinel Lymph Node Biopsy after Neoadjuvant Chemotherapy in Breast Cancer Patients. Cancer Res Treat 2018; 50: 801-812. https://doi. org/10.4143/crt.2017.210 